\title{
Autonomic Job Scheduling Policy for Grid Computing
}

\author{
J.H. Abawajy \\ School of Information technology, \\ Deakin University, Geelong, VIC, 3217, Australia
}

\begin{abstract}
Autonomic middleware services will play an important role in the management of resources and distributed workloads in emerging distributed computing environments. In this paper, we address the problem of autonomic grid resource scheduling and propose a scheduling infrastructure that is capable of self-management in the face of dynamic behavior inherent to this kind of systems.
\end{abstract}

\section{Introduction}

Grid computing is a network of geographically distributed heterogeneous and dynamic resources spanning multiple administrative domains 5 . Grids can potentially furnish large computational and storage resources to solve large-scale problems. However, the need to integrate many independent and heterogeneous subsystems into a well-organized virtual distributed systems introduces new levels of complexity making the underlying Grid environment inherently large, complex, heterogeneous and dynamic. Also it makes the systems highly susceptible to a variety of failures. Some of these failures include node failure, interconnection network failure, scheduling middleware failure, and application failure. Due to the inherent complexity and vulnerabilities, achieving large-scale distributed computing in a seamless manner on Grid computing systems introduces not only the problem of efficient utilization and satisfactory response time but also the problem of fault-tolerance 1 .

One way to address these problems is to make Grid middleware technologies to embrace the concept of self-configuring systems that are able to act autonomously and adapt to changes in application or user needs. There is a synergy towards designing and building computing systems that are capable of running themselves, adjusting to varying circumstances, and preparing their resources to handle most efficiently the workloads we put upon them [7]. This new frontier of designing and building next generation computing systems has become known as autonomic computing [7. The overall goal of autonomic computing is to make systems anticipate needs and allow users to concentrate on what they want to accomplish. The question is then how to design and develop autonomic grid resource management infrastructure that is capable of self-management (i.e., selfcontrol, self-healing, self-configuration, self-optimization, and self-protection) in the face of dynamic behavior inherent to this kind of systems? 
Within this broad problem area, we address the problem of autonmous Grid resource scheduling in the presence of a variety of failures. By autonomous Grid resource scheduling we mean a schediling infrastructure that is capable of acting autonomously and adapt to changes in application or resource failures. We propose an autonomic scheduling infrastructure that is capable of proactively detect and rectify potential faults as applications are executing.

The rest of the paper is organized as follows. In Section 2, related work is presented. This section also establishes the fact that, to a large extent, the problem considered in this paper has not been fully addressed in the literature. Section 3 presents the proposed autonomic scheduling policy. Finally, Section 4 presents the conclusion and future directions.

\section{Related Work}

The human body is self-healing. For example, broken bones mend, and cuts heal. The concept of developing the next generation of computing systems should be driven by the conceptual similarity between biological systems and digital computing systems [6]. Hence, the objectives of autonomic computing is to build computing systems and services that are capable of managing themselves; that can anticipate their workloads and adapt their resources to optimize their performance. 6] [7].

Enhancing the core services of a Grid middleware technologies with autonomic capabilities so that the functions are self-managing is an important research area. There has been some work towards autonomic grid computing 8] 11 6] 9. Liu, and Parashar [11 present an environment that supports the development of self-managed autonomic components, dynamic and opportunistic composition of these components using high-level policies to realize autonomic applications, and provides runtime services for policy definition, deployment and execution. In [6, an autonomic architecture to achieve automated control and management of networked applications and their infrastructure based on XML format specification is presented.

Our main focus in this paper is on autonomic grid resource scheduling middleware. Existing approaches statically allocate or release resources to Grid applications. Moreover, although fault-tolerance is one of the desirable properties of any grid scheduling algorithm, unfortunately it has not been factored into the design of most existing scheduling strategies for Grid computing systems. Research coverage of fault tolerant scheduling is limited as the primary goal for nearly all scheduling algorithms developed so far has been high performance by exploiting as much parallelism as possible. Achieving integrated scheduling and fault-tolerance goal is a difficult proposition as the job scheduling and faulttolerance are difficult problems to solve in their own right.

Clearly, there is a need for a fundamental change in how scheduling middleware for the next generation Grid computing are developed and managed. We believe that the ability to self-manage while effectively exploiting the variably sized pools of resources in an scalable and transparent manner must be 
an integral part of Grid computing scheduling middleware. We are not aware of any work that is currently concentrate on autonomic Grid resource management in general and scheduling middleware in particular. To this end, we propose a scheduling infrastructure that is capable of dynamically scheduling resources while at the same time capable of self-heal to various types of failures.

\section{Autonomic Scheduling Middleware Infrastructure}

In this section, we discuss the proposed autonomic scheduling policy based on a hierarchical approach shown in Figure 1. Note that hierarchical scheduling policies have been used in various platforms such as cluster computing [2] and grid computing [4].

\subsection{System Architecture}

The core system architecture is designed around L-levels of virtual hierarchy, which we refer to as a virtual organization tree, as shown in Figure 1. At the top of the virtual organization tree, there is a system scheduler while at the leaf level there is a local scheduler $(L S)$ for each node. In between the system scheduler and the local schedulers, there exists a hierarchy of middle schedulers $(C S)$.

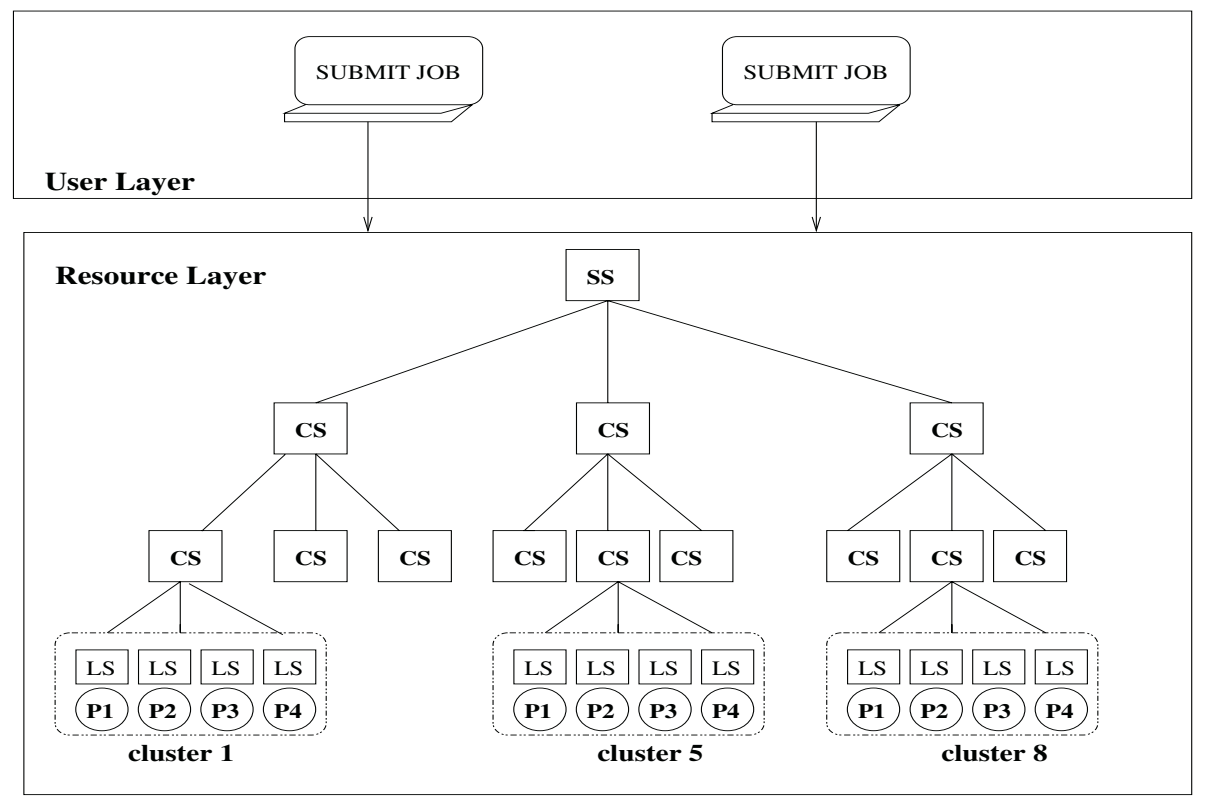

Fig. 1. An example of virtual organization tree (SS: System scheduler; CS: middle scheduler; LS: Local scheduler; and $P_{i}$ : node $i$ ) 
We refer to all processors reachable from a given node in the virtual organization tree as its partition-reach. We associate a parameter called base load level with each node in the virtual organization tree. For non-leaf nodes, the base load level is set to zero. For all the leaf-level nodes, the base load level is the same as the multiprogramming level (MPL) of the node. The MPL parameter of a node controls the maximum number of tasks that can concurrently execute at any given time [2].

\subsection{Autonomic Scheduling Middleware}

Within the hierarchical structure shown in Figure 1, an autonomic Grid resource scheduler can be viewed as a collection of autonomic schedulers that can manage their internal behaviors and their relationships and interactions with other schedulers and the system. The Autonomic Scheduling (ASP) policy automatically replicats jobs and tasks over several grids and processors, keep track of the number of replicas, instantiate them on-demand and delete the replicas when the primary copies of the jobs and tasks successfully complete execution. In this paper, we assume that every middle scheduler in the system is reachable from any other middle scheduler unless there is a failure in the network or the node housing the cluster scheduler. A scheme to deal with node, scheduler and link failures is discussed in 3] 4. Also, without lose of generality, we assume that all incoming jobs are submitted to the system scheduler where they are placed in the job wait queue until a placement decision is made. Figure 2 shows the pseudo-code of the ASP policy. As shown in Figure 2, the ASP policy has three main components namely; Self-scheduling, Job and Task Scheduling and Fault Management components. These components are described in detail in the following subsection.

\subsection{Self-scheduling}

ASP is demand-driven where nodes in the system look for work when their load is below a given threshold. Specifically, whenever the current load level of a nonroot node in the cluster tree falls below its base load level, the node sends a Request for Computation (RFC) message asking for $\mathbf{T}_{\text {req }}$ units of computation to its parent. After sending RFC message to its parent, the node updates its base load level to ensure that it can have only one outstanding RFC at any given time. When a parent with no unscheduled work receives a RFC from a child, if there is a pending RFC at the parent, the new RFC is backlogged and processed when work becomes available. Otherwise, the RFC recursively ascends the cluster tree until the RFC reaches either the system scheduler or a node that has unassigned jobs. In the later case, a set of jobs/tasks are transferred down the hierarchy along the path the RFC has traveled. This amount is determined dynamically during parent and child negotiations and the number of unscheduled jobs as described in the following section. 


\section{SHS algorithm}

1. Self-Scheduling

(a) IF Current load level falls below base load level

$$
T_{r e q}=\text { base load level }- \text { current load. }
$$

(b) Update base load level

\section{ENDIF}

2. Job/Task Assignment

(a) Determine ideal number of jobs/tasks that can be scheduled

$$
T_{\text {target }}=\left\lceil T_{r} \times \text { number of tasks queued }\right\rceil
$$

where $T_{r}$ is the transfer factor and is computed as follows:

$$
T_{r}=\frac{\text { partition-reach of the child node }}{\text { partition-reach of the parent node }}
$$

(b) Adjust the number of jobs that will actually be transferred down one level to the child as follows:

$$
T_{\text {target }}= \begin{cases}\min \left(T_{r e q}, \text { number of tasks queued }\right) & \text { if } T_{r e q}>T_{\text {target }} \\ \min \left(T_{r e q}, \text { child partition-reach }\right) & \text { Otherwise. }\end{cases}
$$

where $T_{r e q}$ is the is the number of computations requested by the child scheduler.

(c) Finally, if the target size determined in Equation 2 is smaller than the $T_{r e q}$ from the child, then the parent will send the lesser of $T_{r e q}$ and the number of tasks queued. Otherwise, the set of tasks sent to the child is the lesser of $T_{\text {target }}$ and child partition-reach.

(d) Send a replica of the job to each site and update job table and backup scheduler.

3. Monitor the job execution

FOR replica-interval DO

(a) Prompt all remote SMs for job status

(b) Determine the number of healthy replicas (i.e., $h$ )

(c) IF any replica is done THEN

i. Tell all remote SMs to terminate their replica

ii. Update job table

(d) ELSEIF $(h>n)$ THEN

i. Select last replica from set to terminate

ii. Update job table

(e) ELSE

i. Pick next site from set to terminate

ii. Inform the remote SM to execute the job

iii. Update job table

ENDIF

ENDFOR

Fig. 2. Self-healing scheduling algorithm 


\subsection{Job and Task Placement}

First, we determine an ideal number of jobs/tasks that can possible be sent to a child scheduler as shown in Equation 2. Once the target number, $T_{\text {target }}$, of jobs that can possibly be transferred to a child is determined, the algorithm then considers the size of the RFC from the child as a hint to adjust the number of jobs that will actually be transferred down one level to the child. Finally, if the target size is smaller than the $T_{r e q}$ from the child, then the parent will send the lesser of $T_{r e q}$ and the number of tasks queued. Otherwise, the set of tasks sent to the child is the lesser of $T_{\text {target }}$ and child partition-reach. Note that this is a dynamic load distribution algorithm that changes the size of the batch at run time, allocating large size to larger clusters while smaller clusters are allocated small size.

With respect to which jobs are dispatched, ASP favors jobs that have their replicas within the partition reach of the requesting schedulers. If there are no such jobs, then jobs belonging to the left sibling of the requesting node is searched. If this fails the jobs of the right sibling of the requesting node are selected. This process continues until the exact number of jobs to be sent to the requesting node is reached. The motivation for this job selection scheme is that we minimize replica management overheads (e.g., the replica instantiation latency) in case the original job fails. We also reduce the job transfer latency as we have to only send control messages to the child scheduler if the replica is already located there. Finally, it reduces the time that a child scheduler waits for the jobs to arrive, which increases system utilization. After dispatching the jobs to a child, the parent informs the backup scheduler about the assignment and then updates the application status table (AST) to reflect the new assignment.

\subsection{Failure Management}

A fail-over strategy is used when a link or a node failure is detected. Link failure is addressed by rerouting traffic from the failed part of the network to another portion of the network. For non-root nodes, the child scheduler is informed to communicate through its closest sibling of the parent. If the failed node is the root, then we choose the closest functional and reachable sibling. When the failed link is repaired, traffic is rerouted over the primary route. Similarly, when a node is repaired, if the node used to be the primary scheduler, then the node that replaced it is told to send to the recovered node and all former children are also notified to update their parent id. The node then rejoins the system and provides services immediately after recovery. If it was a backup node, recovery is not necessary. A detailed discussion of the fail-over strategy is given in [3] [4]

Job Replication. The replica creation and placement is to ensures that a job and its constituent task are stored in a number of locations in the cluster tree. The policy maintains some state information for failure and recovery detections in Application Status Table (AST). Jobs are replicted over clusters while tasks are replicated over processors. Specifically, when a job with fault-tolerance requirement arrives into the system, SHS undertakes the following steps: (1) create 
a replica of the job; (2) keep the replica and send the original job to a child that is alive and reachable; and (3) update the application status table (AST) to reflect where the job replicas are located. This process recursively follows down the cluster tree until we reach the lowest level cluster scheduler (LCS) at which point the replica placement process terminates.

Replica Monitoring. The SHS monitors applications at job-level (between non-leaf nodes and their parents) and at task-level (between leaf nodes and their parents). A monitoring message exchanged between a parent and a leaflevel node is called a report while that between non-leaf nodes is called a summary. A report message contains status information of a particular task running on a particular node and sent every REPORT-INTERVAL time units. In contrast, the summary message contains a collection of many reports and sent every SUMMARY-INTERVAL time periods such that REPORT-INTERVAL < SUMMARY-INTERVAL.

When a processor completes execution of a task, the report message contains a FINISH message. In this case, the receiving scheduler deletes the corresponding replica and informs the backup scheduler to do the same. When the last replica of a given job is deleted, the job is declared as successfully completed. In this case, the cluster scheduler immediately sends a summary message that contains the COMPLETED message to the parent scheduler, which deletes the copy of the job and forward the same message to its parent. This process continues recursively until all replicas of the job are deleted.

Failure Detection and Recovery. After each assignment, the children periodically inform their parents the health of the computations as discussed above. If the parent does not receive any such message from a particular child in a given amount of time, then the parent suspects that the child has failed. In this case, it notes this fact in the AST and sends a request for report message to the child. If a reply from the child has not been received within a specific time frame, the child is declared dead. When a failure is detected, a recovery procedure is initiated to handle the failure. The recovery mechanism restarts a replica of the failed primary task as soon as possible.

\section{Conclusion and Future Directions}

In this paper, we presented a scalable framework that loosely couples the dynamic job scheduling approach with the hybrid (i.e., passive and active replications) approach to schedule jobs efficiently while at the same time providing fault-tolerance. The main advantage of the proposed approach is that fail-soft behaviour (i.e., graceful degradation) is achieved in a user-transparent manner. Furthermore, being a dynamic algorithm estimations of execution or communication times are not required. An important characteristic of our algorithm is that it makes use of some local knowledge like faulty/intact or busy/idle states of nodes and about the execution location of jobs. Another important charac- 
teristic of the proposed approach is that they are applicable for a wide variety of target machines including Grid computing.

We are currently implementing and studying the performance of the proposed policy. In the proposed self-healing distributed framework, the latency of detecting the errors might be affected by message traffic in the communication network. To address this problem, we intend to develop an on-line mechanism to dynamically measure the round-trip time of the underlying network and calculate the error latency accordingly.

Acknowledgement. I appreciate the help of Maliha Omar without whose help this paper would not have been realized.

\section{References}

1. J. H. Abawajy. Fault-tolerant scheduling policy for grid computing systems. In Proceedings of IEEE 18th International Parallel $\&$ Distributed Processing Symposium (IPDPS04), pages 238-146, 2004.

2. Jemal H. Abawajy and Sivarama P. Dandamudi. Parallel job scheduling on multicluster computing systems. In Proceedings of IEEE International Conference on Cluster Computing (CLUSTER'03), pages 11-21, 2003.

3. Jemal H. Abawajy and Sivarama P. Dandamudi. A reconfigurable multi-layered grid scheduling infrastructure. In Hamid R. Arabnia and Youngsong Mun, editors, Proceedings of the International Conference on Parallel and Distributed Processing Techniques and Applications, PDPTA '03, June 23 - 26, 2003, Las Vegas, Nevada, USA, Volume 1, pages 138-144. CSREA Press, 2003.

4. Jemal H. Abawajy and Sivarama P. Dandamudi. Fault-tolerant grid resource management infrastructure. Journal of Neural, Parallel and Scientific Computations, 12:208-220, 2004.

5. Ian T. Foster, Carl Kesselman, and Steven Tuecke. The anatomy of the grid enabling scalable virtual organizations. CoRR, cs.AR/0103025, 2001.

6. Salim Hariri, Lizhi Xue, Huoping Chen, Ming Zhang, Sathija Pavuluri, and Soujanya Rao. Autonomia: An autonomic computing environment. In IEEE International Performance Computing and Communications Conference, 2003.

7. Paul Horn. Autonomic computing. The Economist print edition, September 19, 2002.

8. Gang HUANG, Tiancheng LIU, Hong MEI, Zizhan ZHENG, Zhao LIU, and Gang FAN. Towards autonomic computing middleware via reflection. In COMPSAC, 2004.

9. Gail Kaiser, Phil Gross, Gaurav Kc, Janak Parekh, and Giuseppe Valetto. An approach to autonomizing legacy system. In IBM Almaden Institute Symposium, 2002.

10. Jeffrey O. Kephart and David M.Chess. The vision of autonomic computing. IEEE Computer, pages 41-50, 2003.

11. H. Liu and M. Parashar. A component based programming framework for autonomic applications. In In Proceedings of the International Conference on Autonomic Computing, 2004. 